\title{
Creation of an Antiferromagnetic Exchange Spring
}

\author{
A. Scholl, ${ }^{1}$ M. Liberati, ${ }^{1,2}$ E. Arenholz, ${ }^{1}$ H. Ohldag,,${ }^{1,3}$ and J. Stöhr ${ }^{3}$ \\ ${ }^{1}$ Lawrence Berkeley National Laboratory, Berkeley, CA 94720, USA \\ ${ }^{2}$ INFM - National Center on nanoStructures and bioSystems at Surfaces (S3), Via Campi 213/a, 41100-Modena, Italy \\ ${ }^{3}$ Stanford Synchrotron Radiation Laboratory, Stanford, CA 94309, USA
}

(Dated: April 6, 2004)

\begin{abstract}
We present evidence for the creation of an exchange spring in an antiferromagnet due to exchange coupling to a ferromagnet. X-ray magnetic linear dichroism spectroscopy on single crystal $\mathrm{Co} / \mathrm{NiO}(001)$ shows that a partial domain wall is wound up at the surface of the antiferromagnet when the adjacent ferromagnet is rotated by a magnetic field. We determine the interface exchange stiffness and the antiferromagnetic domain wall energy from the field dependence of the direction of the antiferromagnetic axis, the antiferromagnetic pendant to a ferromagnetic hysteresis loop. The existence of a planar antiferromagnetic domain wall, proven by our measurement, is a key assumption of most exchange bias models.
\end{abstract}

Antiferromagnetic materials play an important role in technology and in basic sciences. However, the vanishing magnetization caused by their compensated magnetic structure renders them a difficult class of materials to study. Particularly interesting effects occur at the boundary of an antiferromagnet (AFM) and a ferromagnet (FM). A prominent example is exchange bias, which was discovered half a century ago [1] and which is used today in magneto-electronic devices to pin the magnetization of a ferromagnetic layer (for a review see [2-4]). Using x-ray spectroscopy we now have the ability to investigate the microscopic structure of these fascinating systems right at the interface. In this Letter we show that an exchange-coupled ferromagnet/antiferromagnet system behaves like an antiferromagnetic exchange spring magnet, very similar to ferromagnetic spring magnets that consist of coupled soft- and hard-magnetic ferromagnets [5-7]. Like in a ferromagnetic spring magnet, a planar domain wall is wound up in the antiferromagnet when the magnetization of the ferromagnet is rotated or switched, as illustrated in Fig. 1. The existence of such a wall is a key assumption of models describing exchange bias, but it has never been confirmed experimentally [24, 8-12].

We chose $\mathrm{Co}$, a ferromagnet, on $\mathrm{NiO}$, an antiferromagnet, for our study, since it is an extensively studied exchange bias system and since $\mathrm{NiO}$ single crystals have a well-understood magnetic bulk and interface structure $[13,14]$. It is known that exchange bias leads to a change in the $\mathrm{NiO}$ domain population [15]. $2.5 \mathrm{~nm}$ Co was grown by e-beam evaporation at room temperature under UHV conditions onto freshly cleaved $\mathrm{NiO}(001)$ single crystal surfaces, following a recipe described in [14], and was capped with $2 \mathrm{~nm} \mathrm{Pd}$ to prevent oxidization. Co and $\mathrm{NiO}$ are strongly exchange coupled, as shown by their similar Photoemission Electron Microscopy (PEEM) domain images in Fig. 2. The coupling is believed to be mediated by uncompensated $\mathrm{Ni}$ spins that originate from a chemical reaction at the $\mathrm{Co} / \mathrm{NiO}$ interface [16]. In the $\mathrm{NiO}$ image two classes of interface domains are distinguished by in-plane AFM axes along [011] and [01̄]]. The magnetization in Co domains is aligned with the AFM axis of the $\mathrm{NiO}$ substrate, which is a remarkable result, since it implies a parallel coupling between a compensated AFM surface and a FM [14].

The magnetic hysteresis of the Co magnetization and its effect on the $\mathrm{NiO}$ magnetic structure were investigated by x-ray magnetic dichroism spectroscopy using total electron yield detection. X-ray magnetic dichroism (XMD) is an x-ray absorption technique that is sensitive to magnetic moments and moment orientations of FMs and AFMs. XMD is element-specific, quantitative, and, using the total electron yield, surface sensitive with a probing depth of 2-5 nm. Surface sensitivity allows us to focus on the magnetic structure of the Co layer and of $\mathrm{NiO}$ close to the $\mathrm{Co} / \mathrm{NiO}$ interface [16]. The rotation of the top of the AFM domain wall is probed because the probing depth is small compared to the $\mathrm{NiO}$ domain wall width. Magnetic fields of up to $0.8 \mathrm{~T}$ were applied using an octupole electromagnet that allows rotation of the magnetic field in any direction. The spectroscopy experiments were conducted at the elliptically polarizing undulator beamline 4.0.2 of the Advanced Light Source. The x-ray spot size was $1 \mathrm{~mm}$ x $0.1 \mathrm{~mm}$.

The Co hysteresis loop shown in Fig. 2 was acquired using x-ray magnetic circular dichroism (XMCD) at a $30^{\circ}$ angle of incidence along [011], which is also the field direction. XMCD detects the angle between the direction of the magnetization and the helicity vector of the circular x-ray polarization. The loop can be understood as a superposition of a hard-axis loop and an easy-axis loop. The easy-axis loop originates from Co domains that are coupled to [011] NiO interface domains. The hardaxis loop originates from domains that possess a uniaxial anisotropy perpendicular to [011] through coupling to $[01 \overline{1}] \mathrm{NiO}$ interface domains. When the magnetization of these Co domains is forced by the magnetic field into the [011] direction, a torque is created in the $\mathrm{NiO}$ through exchange coupling, which should wind up a planar wall. $\mathrm{NiO}$ x-ray magnetic linear dichroism (XMLD) spectra in 
Fig. 3 substantiate this hypothesis. XMLD detects the angle between the antiferromagnetic axis and the linear x-ray polarization, averaged over the x-ray spot. The spectra were measured with the linear x-ray polarization along the $[01 \overline{1}](\mathrm{z})$ direction in normal incidence. In-plane fields of $0.7 \mathrm{~T}$ were applied along the [011] (y) and the $[01 \overline{1}]$ (z) directions to saturate the magnetization of the Co layer. We indeed observe a linear dichroism of the AFM between these geometries. A field applied along $\mathrm{z}$ parallel to the $\mathrm{x}$-ray polarization leads to a decrease of the lower-energy $\mathrm{Ni} L_{2}$ peak and an increase of the higher-energy peak, commensurate with a rotation of the y AFM domains into z. A field applied along y leads to the opposite dichroism, commensurate with a rotation of the $\mathrm{z}$ AFM domains into $\mathrm{y}$. The observed rotation parallel to the field rules out a spin flop of the AFM from the direct interaction with the field. The XMLD difference spectrum of uncoated $\mathrm{NiO}(001)$ also shows no rotation, Fig. 3. Thus the FM acts as a lever that by magnetic exchange pulls the magnetic moments of the AFM with it when an external field is applied. A domain wall is wound up.

The $L_{2}$ peak ratio quantifies the angle of rotation as a function of the applied field: $R_{L_{2}}=a-b * \cos ^{2} \varphi$, where $a>b>0$ are material-dependent constants [17]. $\varphi$ is the angle between the AFM axis $\boldsymbol{A}$ and the linear x-ray polarization $\boldsymbol{E}$. A plot of the peak ratio as function of the applied field is the AFM pendant of a FM hysteresis loop. The AFM loop quantifies the response of the antiferromagnetic spring to an external field. One branch of a loop is shown in Fig. 4, measured in two geometries. In the perpendicular geometry, the loop was acquired with the field $\boldsymbol{H}$ applied perpendicular to the x-ray polarization $\boldsymbol{E}$ (open squares). In the parallel geometry, the loop was acquired with $\boldsymbol{H}$ and $\boldsymbol{E}$ aligned (filled squares). Pairs of loops with the x-ray polarization parallel to [011] and $[01 \overline{1}]$ were averaged in order to suppress artifacts caused by the possible imbalance of the areas of the two NiO interface domains within the x-ray spot. Furthermore, the four ascending and descending branches for positive and negative fields were averaged, since they showed negligible hysteresis at high fields. The increasing $L_{2}$ ratio in the perpendicular geometry indicates an increasing angle between the AFM axis and x-ray polarization. The ratio approaches $R_{L_{2}}^{\max }=1.27$, which is indicative of an approximately perpendicular orientation [17]. In the parallel geometry, the ratio decreases but does not quite reach $R_{L_{2}}^{\text {min }}=0.82$, a value which would indicate a parallel orientation of AFM axis and polarization. We will discuss the asymmetric shape of the two branches later. The wall rotation angle $\alpha$ (see Fig. 4) can be approximated by normalizing the difference between the measured $L_{2}$ ratios $R_{L_{2}}^{\perp}-R_{L_{2}}^{\|}$to $R_{L_{2}}^{\max }-R_{L_{2}}^{\min }=0.45$, which is the expected maximum variation between a parallel and a perpendicular state in $\mathrm{NiO}[17]: \sin ^{2} \alpha=\left(R_{L_{2}}^{\perp}-R_{L_{2}}^{\|}\right) / 0.45$.
Our observations confirm the existence of a antiferromagnetic wall in a coupled FM/AFM system conjectured by Mauri et al. [9], based on an earlier model by Nèel [8]. Mauri's model, originally designed to quantitatively explain exchange bias, assumes a coherent rotation of the FM coupled to an AFM spring, forming a planar wall that reaches into and is anchored in the infinitely thick AFM, as shown in Fig. 1. The rotation angle $\alpha$ of the interface layer of the AFM and the rotation angle $\beta$ of the FM can be determined by minimizing the dimensionless energy functional

$$
\delta=(1-\cos \alpha)+\lambda(1-\cos (\alpha-\beta))-\kappa(1-\sin \beta)
$$

with respect to $\alpha$ and $\beta$. The three terms correspond to the AFM wall energy, the interface exchange energy, and the Zeeman energy, normalized to the energy of a $90^{\circ}$ AFM wall $2 \sqrt{A K}$. The parameters $\lambda=A_{12} / \xi 2 \sqrt{A K}$ and $\kappa=H M d / 2 \sqrt{A K}$ are the interface exchange energy and the Zeeman energy of the FM in a field $\mathrm{H}$, normalized to the wall energy. The field is applied perpendicular to the anisotropy axis of the AFM. We disregard the anisotropy energy of the FM, which we assume is small compared to the interface and wall energies. This is a valid assumption for a polycrystalline, strongly interface-coupled, thin film. We also assume that the AFM anisotropy is uniaxial and that the rotation is in-plane and reversible.

Using $\lambda$ and $\kappa$ as fit parameters, a solution was found numerically that minimizes equation (1) while optimally fitting the measured wall angle $\alpha$, as shown in Fig. 4. An optimal fit was obtained for $\lambda=1.53$ and $\kappa=5.29$, a wall energy $2 \sqrt{A K}=0.66 \mathrm{~mJ} / \mathrm{m}^{2}$, and an interface exchange stiffness $A_{12}=2.1 \cdot 10^{-13} \mathrm{~J} / \mathrm{m}$ [18]. The model describes the data excellently from high fields down to about $50 \mathrm{mT}$, close to the coercivity of the FM, where the switching of the easy-axis domains starts to contribute to the AFM loop.

Our estimate of the wall energy is consistent with the value $0.42 \mathrm{~mJ} / \mathrm{m}^{2}$ [19], which was obtained by microscopy of the width of vertical walls on a similarly prepared $\mathrm{NiO}(001)$ sample. Our estimate is too large by an order of magnitude compared to the result of a torque magnetometry measurement on a strain-free and single domain crystal [20]. We attribute this deviation to the high strain and defect density of our mechanically cleaved crystals. It is known, that completely strain-free samples have a significantly reduced magnetic coercivity $[13,20]$. The large ratio $\lambda$ between the coupling energy and the domain wall energy explains why $\mathrm{NiO}$ single crystals show poor exchange bias properties. Exchange bias requires that the AFM wall energy or anisotropy energy be larger than the interface coupling energy, in order to obtain a stable domain state in the AFM (for a review see [2-4]).

The measured interface exchange stiffness, which is approximately $1 \%$ of the exchange stiffness of $\mathrm{Ni}$ metal, is reasonable in magnitude, since its value sets an upper limit for the maximum bias field of a system. Typically, 
bias fields amount to much less than $1 \%$ of the exchange field of a fully uncompensated interface because of the small fraction of uncompensated interface spins that pin the magnetization [21].

Finally we will discuss the remarkable asymmetry between the two branches of the AFM loop, measured with $\boldsymbol{E} \| \boldsymbol{H}$ and $\boldsymbol{E} \perp \boldsymbol{H}$. So far we have assumed that the rotation plane of the $\mathrm{NiO}$ moments is parallel to the sample surface. In that case, however, the variation of the $L_{2}$ ratios $R_{L_{2}}^{\perp}$ and $R_{L_{2}}^{\|}$should be equal [22]. To resolve this discrepancy, we need to take into account that the magnetic anisotropy of $\mathrm{NiO}$ is lowest by far for spin rotations within $\{111\}$ planes $[11,20]$. Therefore, spins preferentially rotate within $\{111\}$ planes and avoid rotations out of these planes. Fig. 5 illustrates this idea. We consider a $[0 \overline{1} 1]$-oriented domain within the (111) plane, which is subjected to a field along the perpendicular $[0 \overline{1} \overline{1}]$ direction. The high anisotropy between $\{111\}$ planes prevents the $\mathrm{NiO}$ moments from leaving the (111) plane; the moments therefore rotate within that plane into a direction that minimizes the angle to the magnetization of the

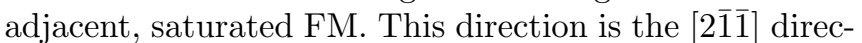
tion. We have modeled the $L_{2}$ ratio in the parallel $\boldsymbol{E} \| \boldsymbol{H}$ and the perpendicular $\boldsymbol{E} \perp \boldsymbol{H}$ geometry as a function of the angle $\alpha$ of the AFM axis to the initial [011] direction [23]. The result is plotted at the bottom of Fig. 5. The variation in the $L_{2}$ ratio is now indeed asymmetric, since the AFM axis reaches a perpendicular orientation to $[0 \overline{1} 1]$ but is never fully aligned with the direction of the applied field. An improved model of the wall rotation should take into account the non-planar rotation of the $\mathrm{NiO}$ moments and the non-collinear coupling between these and the Co moments. Such a model is beyond the scope of this paper.

In summary, we have observed the creation of a planar, antiferromagnetic domain wall in the system $\mathrm{Co} / \mathrm{NiO}$. The existence of such a planar wall is a fundamental assumption in magnetic models of exchange bias. By measuring ferromagnetic and antiferromagnetic loops we have explored the field-dependent properties of the interface region. Here, x-ray magnetic linear dichroism (XMLD) provided us with the unique ability to directly measure the dynamics of the antiferromagnetic interface. Exposed to a magnetic field, the system reacts like an antiferromagnetic exchange spring magnet. By analyzing the wall rotation we have determined fundamental characteristics of the exchange spring: the antiferromagnetic wall energy and the interface exchange stiffness.

This work was supported by the Office of Basic Energy Sciences, of the U.S. Department of Energy (ALS and
FIG. 1: Creation of an antiferromagnetic exchange spring: FM rotates into field direction and creates a planar domain wall in the exchange-coupled AFM.

FIG. 2: Left: Ferromagnetic hysteresis loop of $2.5 \mathrm{~nm}$ $\mathrm{Co} / \mathrm{NiO}(001)$. The loop was measured as the asymmetry of the $L_{3}$ intensity using opposite elliptical polarization at $30^{\circ} \mathrm{x}-$ ray grazing incidence. Right: PEEM images demonstrate domain correlation and coupling between $\mathrm{NiO}$ and Co. Arrows indicate the $\mathrm{NiO}$ AFM axes and Co magnetization directions.

SSRL). M.L. acknowledges financial support by M.I.U.R. - PNR n. RBNE017XSW. The authors would like to thank H.C. Siegmann for very rewarding discussions.

[1] W. Meiklejohn and C. Bean, Phys. Rev. 102, 1413 (1956).

[2] A. E. Berkowitz and K. Takano, J. Magn. Magn. Mater. 200, 552 (1999).

[3] M. Kiwi, J. Magn. Magn. Mater. 234, 584 (2001).

[4] J. Nogues and I. K. Schuller, J. Magn. Magn. Mater. 192, 203 (1999)

[5] E. F. Kneller and R. Hawig, IEEE Trans. Magn. 27, 3588 (1991).

[6] E. E. Fullerton, J. S. Jiang, M. Grimsditch, C. H. Sowers, and S. D. Bader, Phys. Rev. B 58, 12193 (1998).

[7] K. V. O'Donovan, J. A. Borchers, C. F. Majkrzak, O. Hellwig, and E. E. Fullerton, Phys. Rev. Lett. 88, 067201 (2002).

[8] L. Nèel, Ann. Phys. (Paris) 2, 61 (1967).

[9] D. Mauri, H. C. Siegmann, P. S. Bagus, and E. Kay, J. Appl. Phys. 62, 3047 (1987).

[10] T. C. Schulthess and W. H. Butler, Phys. Rev. Lett. 81, 4516 (1998).

[11] M. D. Stiles and R. D. McMichael, Phys. Rev. B 59, 3722 (1999).

[12] H. W. Xi and R. M. White, Phys. Rev. B 61, 1318 (2000).

[13] W. Roth, J. Appl. Phys. 31, 2000 (1960).

[14] H. Ohldag, A. Scholl, F. Nolting, S. Anders, F. U. Hillebrecht, and J. Stöhr, Phys. Rev. Lett. 86, 2878 (2001).

[15] W. Zhu, L. Seve, R. Sears, B. Sinkovic, and S. S. P. Parkin, Phys. Rev. Lett. 86, 5389 (2001).

[16] H. Ohldag, T. J. Regan, J. Stöhr, A. Scholl, F. Nolting, J. Lüning, C. Stamm, S. Anders, and R. L. White, Phys. Rev. Lett. 87, 247201 (2001).

[17] D. Alders, L. H. Tjeng, F. C. Voogt, T. Hibma, G. A. Sawatzky, C. T. Chen, J. Vogel, M. Sacchi, and S. Ia-

FIG. 3: Left: XMLD spectra and difference at the Ni edge of $\mathrm{Co} / \mathrm{NiO}$ in normal incidence in an in-plane field of $0.7 \mathrm{~T}$ using vertical linear polarization $(\mathrm{z})$. The difference in the $L_{2}$ peak ratio for fields $\mathrm{H}$ along $\mathrm{y}$ and $\mathrm{z}$ indicates a rotation of the AFM axis into the field direction. Bare $\mathrm{NiO}$ does not show an XMLD, ruling out a spin-flop. Right: Schematic drawing of the domain rotation. 
FIG. 4: Quadrant of the NiO AFM hysteresis loop. The $L_{2}$ peak ratio (left abscisse) is shown as a function of field strength perpendicular (open squares) and parallel (filled squares) to the linear x-ray polarization vector. The deduced rotation angle $\alpha$ of the AFM wall is plotted using circles (right abscisse). The fit based on a planar domain wall model (thick line) reproduces the data.

FIG. 5: Top: Rotation of the spin axis (dotted arrows) of a $[0 \overline{1} 1]$ interface domain in a field along $[0 \overline{1} \overline{1}]$. The AFM axis rotates within the (111) plane (dark gray) out of the (100) plane (light gray). Bottom: The calculated $L_{2}$ ratio plotted as a function of the rotation angle $\alpha$. cobucci, Phys. Rev. B 57, 11623 (1998).

[18] We use $d_{C o}=2.5 \mathrm{~nm}, M_{C o}=1.4 \cdot 10^{6} \mathrm{~A} / \mathrm{m}, \xi_{C o-N i}=$ $0.21 \mathrm{~nm}$, which is half the lattice constant of $\mathrm{NiO}$.

[19] N. B. Weber, H. Ohldag, H. Gomonaj, and F. U. Hillebrecht, Phys. Rev. Lett. 91, 237205 (2003).

[20] K. Kurosawa, M. Miura, and S. Saito, J. Phys. C: Solid St. Phys. 13, 1521 (1980).

[21] H. Ohldag, A. Scholl, F. Nolting, E. Arenholz, S. Maat, A. T. Young, M. Carey, and J. Stöhr, Phys. Rev. Lett. 91, 017203 (2003).

[22] The observed asymmetry also rules out that the observed dichroism is due to lateral domain wall motion rather than the creation of a planar wall.

[23] The model takes into account the equal population of four in-plane $\mathrm{NiO}$ (110) domains but keeps the axis fixed in domains in which the moments are already aligned with the field. 
Fig. 1

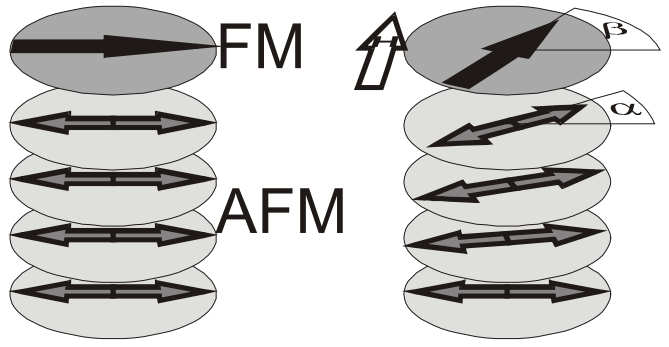


Fig. 2

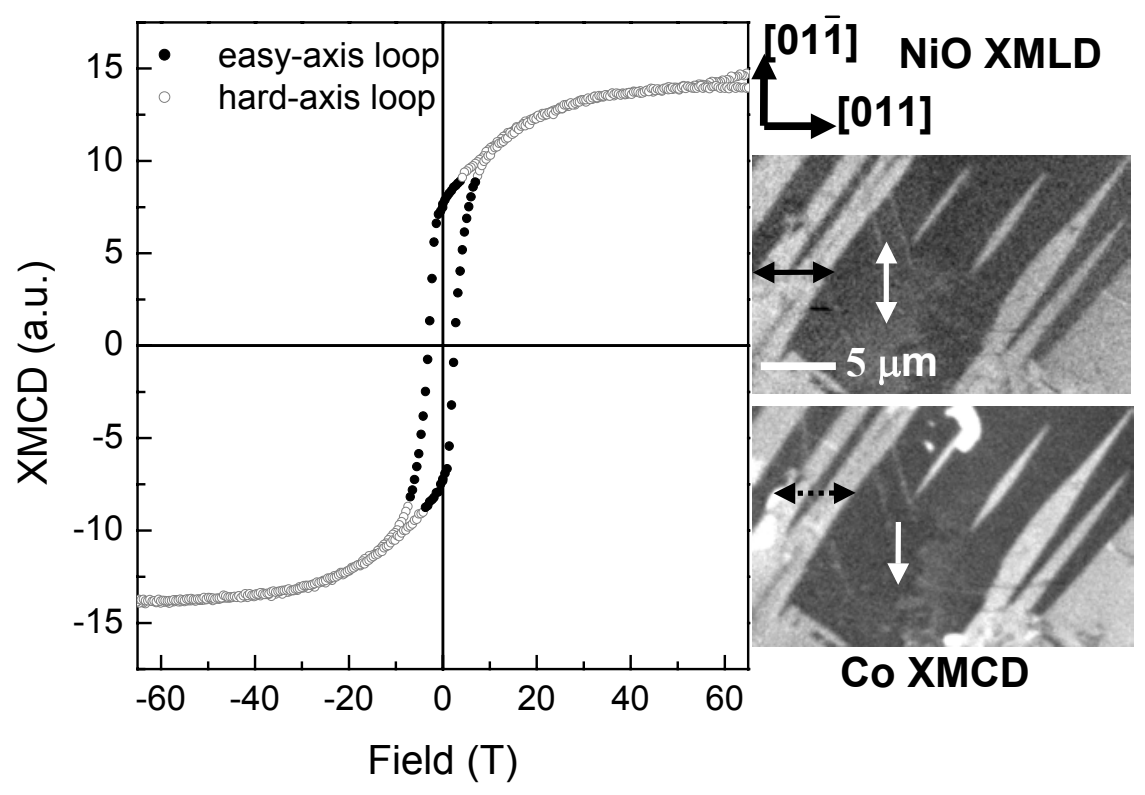


Fig. 3

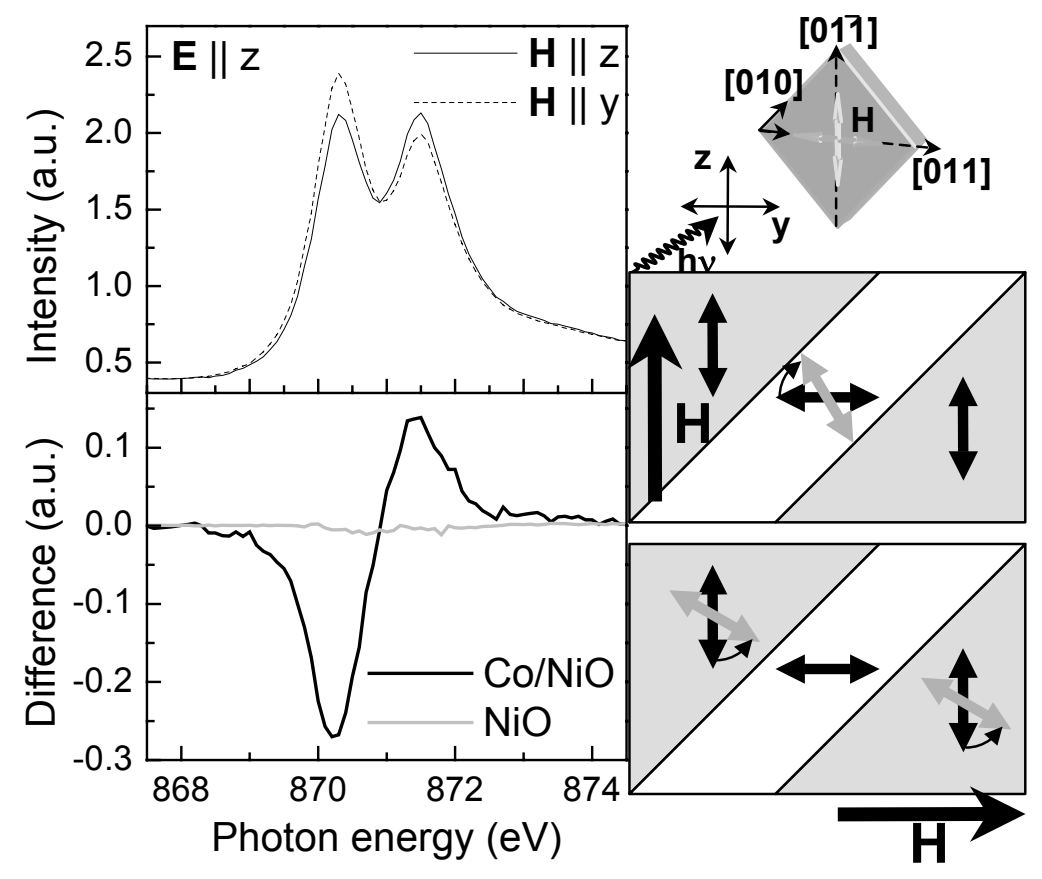


Fig. 4

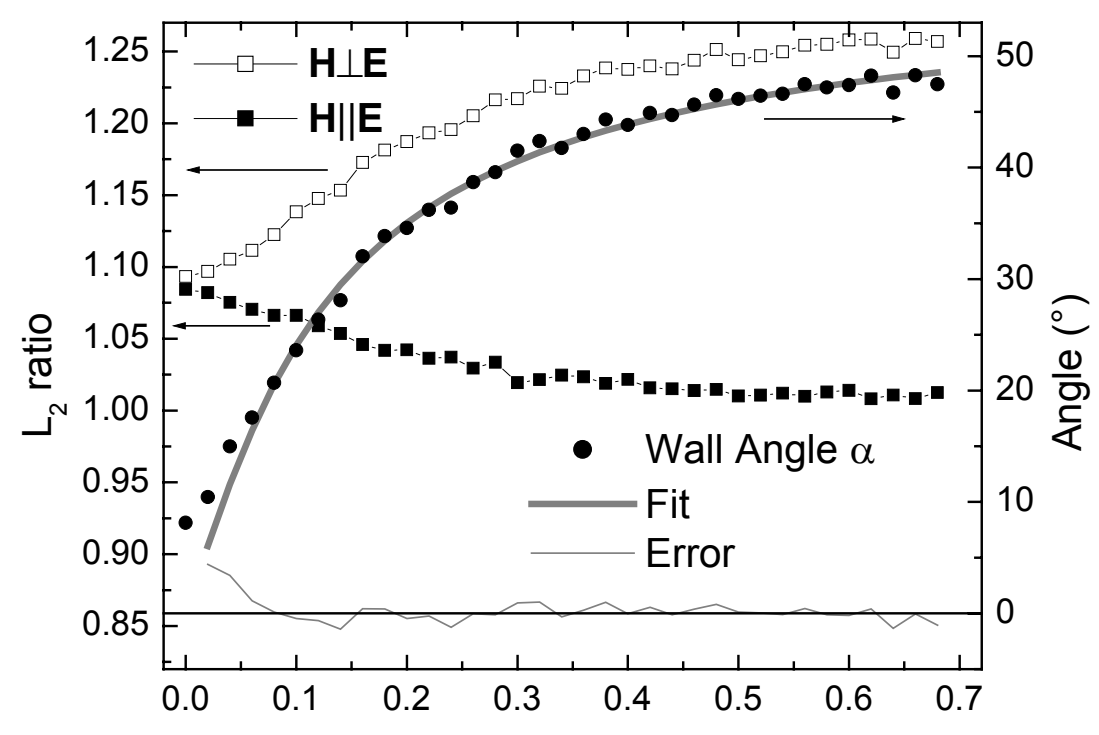

Field ( $\mathrm{T}$ ) 
Fig. 5

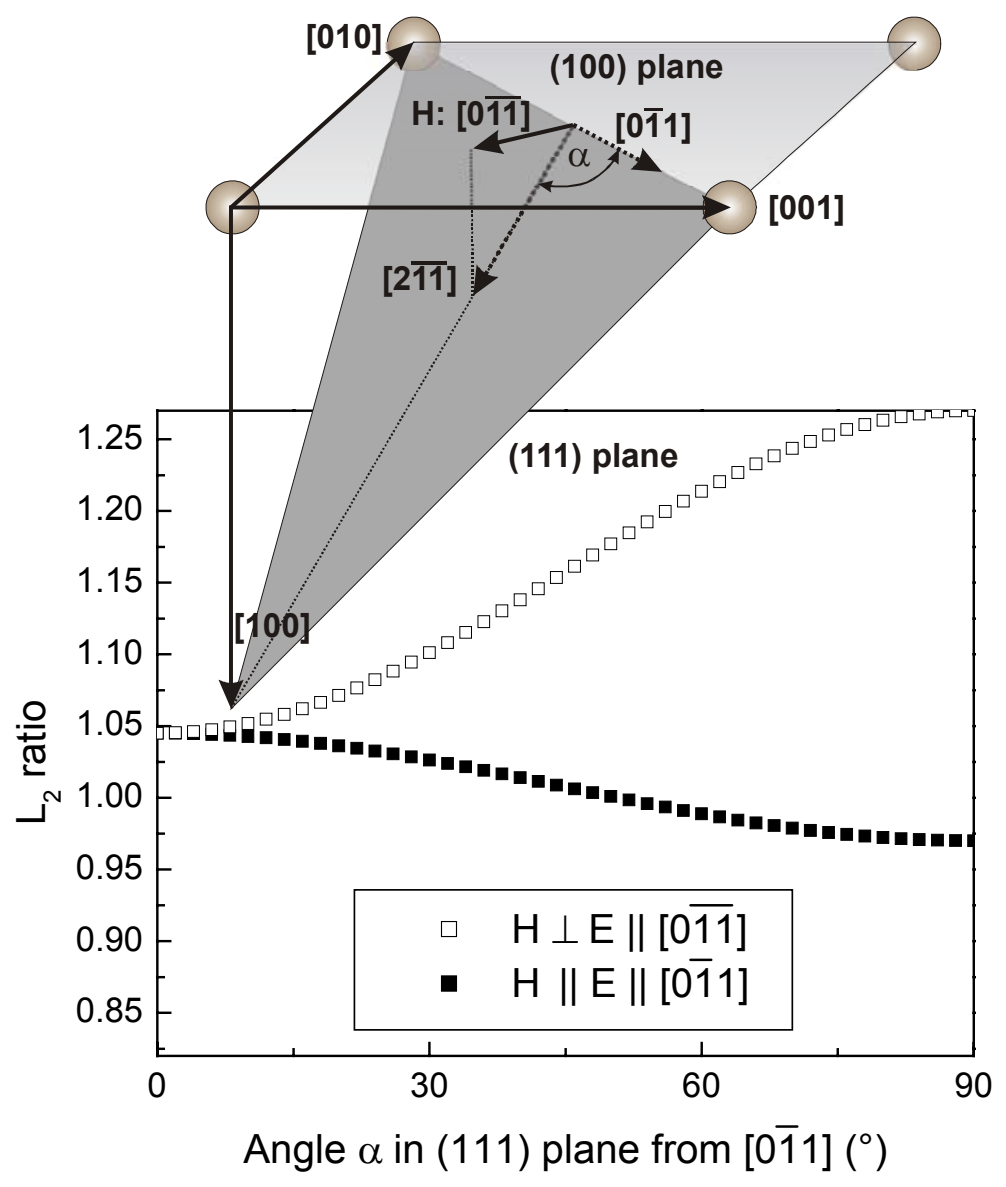

